\title{
FORMACIÓN JURÍDICA, COMPETENCIAS Y MÉTODOS DE ENSEÑANZA: PREMISAS*
}

\author{
Patricio Lazo GonzÁlezZ*
}

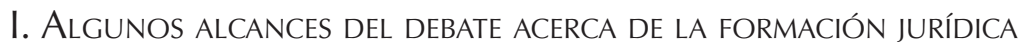

En marzo del año 2009, en cumplimiento de las obligaciones que la ley impone, el entonces Presidente de la Corte Suprema, ministro Urbano Marín, dio cuenta de la labor de los tribunales el año 2008. Dentro del apartado VII, en el punto 4 (p. 13) de su exposición el alto magistrado declaró:

"Es preocupante el número de abogados que se han titulado en el último tiempo. Desde 1997 y hasta 2008 han recibido sus diplomas 15.894 profesionales, a los que se suman los 350 que juraron en enero pasado. Como le corresponde otorgar el título, a la Corte Suprema ${ }^{1}$ le inquieta no sólo la cantidad de profesionales que se han incorporado a la abogacía, sino la calidad de su preparación y los riesgos que un alto número de letrados en competencia representa para la ética profesional. Este problema ha sido y debería seguir siendo objeto de estudio con el Colegio de Abogados, si bien eventualmente requiere de una iniciativa legal". ${ }^{2}$

Dos problemas, por consiguiente, se asocian, en la exposición del entonces Presidente del máximo tribunal nacional: el de la preparación, es decir, el de

\footnotetext{
* Este trabajo es uno de los resultados del proyecto MECESUP ATA 0706 ANF, Rediseñar el plan de estudio de la "Licenciatura en Ciencias Jurídicas" de las Universidades de Atacama y Antofagasta y corresponde a una versión ampliada y con aparato crítico, de la comunicación presentada al Congreso de Decanos de Derecho, organizado por la Universidad de Valparaíso, los días 5 y 6 de agosto de 2006, cuyas actas serán publicadas en los Anales de la ya centenaria Facultad de Derecho de dicha casa de estudios. Colaboración recibida el 9 de marzo y aprobada el 20 de abril de 2011.

** Decano y Profesor asociado de la Facultad de Ciencias Jurídicas y Sociales de la Universidad de Antofagasta; Doctor en Derecho; Profesor de Derecho romano; Historia del Derecho y Filosofía del Derecho. Correo electrónico: patricio.lazo@uantof.cl.

1 Marín, Urbano, "Cuenta pública del Presidente de la Corte Suprema en la inauguración del año judicial 2009", 2009, p. 13. En: http://www.poderjudicial.cl [visitado el 02/02/2011]. A diferencia de lo que ocurre con los demás títulos profesionales, el de abogado no es otorgado por las universidades que imparten la carrera de Derecho, sino por la Corte Suprema. Así lo dispone el art. 21 del Código Orgánico de Tribunales: "El título de abogado será otorgado en audiencia pública por la Corte Suprema reunida en tribunal pleno, previa comprobación y declaración de que el candidato reúne los requisitos establecidos por los artículos 523 y 526".

${ }^{2}$ Marín, "Cuenta pública", cit. nota n. 1, p. 13.
} 
la calidad de la formación de los profesionales y el de los riesgos que correría la ética profesional a raíz de un número excesivo de los mismos. ${ }^{3} \mathrm{~A}$ primera vista, Ilama la atención que la ética profesional esté disociada del problema de la formación, puesto que bien podría entenderse que con más o menos abogados, una deficiente formación ética igualmente pone en riesgo la puesta en práctica de ésta. Con todo, las palabras del ministro Marín no pasaron inadvertidas y dieron origen a un debate, más bien breve y sólo a través de la prensa. En primer término se implicaron en el debate tres decanos, ${ }^{4}$ algunos de los cuales manifestaron su preocupación por la existencia de instituciones universitarias que a la fecha impartían la carrera de Derecho en tres años, poniéndose énfasis, nuevamente, en el problema la calidad de la formación. ${ }^{5}$ En ese mismo artículo de prensa, dos decanos se mostraron partidarios de un examen nacional, idea que conseguiría el patrocinio de otros tres, todos representantes de instituciones universitarias de gran importancia en la tradición chilena de formación jurídica. ${ }^{6}$ Este conjunto de autoridades universitarias decidieron avanzar en el desarrollo de la propuesta, entre cuyas características destacaba su carácter voluntario. ${ }^{7}$ Sin embargo, un año después, la prensa informaba que la iniciativa no había avanzado suficientemente, estancándose en la etapa de conversaciones. ${ }^{8}$

El debate descrito sirve para contextualizar la entrada en escena del Ejecutivo, el que a través del Ministerio de Educación, dio a conocer un documento de trabajo ${ }^{9}$, algunas de cuyas premisas creo importante poner de relieve:

a) Se aprecian aumentos significativos tanto en el número de alumnos de la carrera de Derecho, como en el de titulados. En cuanto a los primeros, las estimaciones para el año 2001 eran 20.034, en tanto que para el año 2009,

\footnotetext{
${ }^{3}$ Similares aprensiones reiteró en su cuenta pública de este año el actual Presidente de la Corte Suprema, Ministro Milton Juica, quien reiteró que la situación actual "requiere una corrección legislativa para la dignidad de la profesión de abogado y por la importancia que reviste la idoneidad de los letrados para asegurar un verdadero Estado de Derecho..." y puso mucho énfasis en el deficiente control ético de la profesión. Vid. Juica, Milton, "Cuenta pública del Presidente de la Corte Suprema en la inauguración del año judicial 2011", 2011. En: http://www.poderjudicial.cl [visitado el 08/03/2011].

${ }^{4}$ Roberto Nahum, de la Facultad de Derecho de la Universidad de Chile, Arturo Yrarrázabal, de la Facultad de Derecho de la Universidad Católica de Chile y Ángela Cattán, de la Facultad de Derecho de la Universidad Central.

${ }^{5}$ El Mercurio (edición nacional) 04.03.2009.

${ }^{6}$ Alejandro Guzmán Brito (Facultad de Derecho de la Pontificia Universidad Católica de Valparaíso), Alberto Balbontín (Facultad de Derecho de la Universidad de Valparaíso) y Sergio Carrasco (Facultad de Derecho de la Universidad de Concepción).

7 El Mercurio (edición nacional) 18.03.2009.

${ }^{8}$ El Mercurio (edición nacional) 21.06.2010.

${ }^{9}$ MinisTeRIO de EdUCACIÓN, "Razones para la exploración de una propuesta de habilitación y certificación de la profesión de abogado en Chile. Documento de Trabajo № 1", 2010.
} 
eran de 33.889 alumnos. En cuanto a los titulados, se destaca que entre 1970 y 2000 hubo 12.163, en tanto que entre 2001 y 2009 hubo 14.980 titulados. El aumento, por consiguiente, ha sido exponencial.

b) Se observa una deficiente evaluación de conocimientos y destrezas que presentan los nuevos abogados, de acuerdo con las siguientes mediciones:

i) Encuestas aplicadas a abogados tutores de la Corporación de Asistencia Judicial. En el ítem "Manejo de conocimientos jurídicos", éstos estimaron que los postulantes tenían una preparación inferior al 70\% de los conocimientos requeridos para la práctica.

ii) En opinión de magistrados de tribunales superiores de justicia, las destrezas exhibidas por los nuevos abogados son deficientes. El desempeño en tribunales, particularmente en alegatos, es percibido por los ministros de distintas cortes como una cualidad decisiva en los litigios.

A la vista de las características del debate que se generó a comienzos de 2009 a partir de la Cuenta del Presidente de la Corte Suprema, es posible concluir que existe una percepción más o menos generalizada, al menos entre los encargados de la formación de abogados, magistrados y del Ejecutivo, acerca de las actuales deficiencias formativas de los profesionales del Derecho, las que estarían concentradas en los siguientes aspectos: conocimientos, destrezas prácticas, consistencia ética. A su vez, estos reproches guardan ciertos puntos de conexión con algunas de las premisas más importantes del movimiento de reformas curriculares inspirado en la formación basada en competencias. ${ }^{10}$ Es decir, algunas de sus críticas (aquellas que se refieren a destrezas, habilidades y a resolución de problemas de índole ética) apuntan directamente hacia el concepto de competencias. Si lo expresamos, entonces, en estos términos, lo que tenemos ante nuestros ojos, junto al problema del déficit del conocimiento, es el de una débil formación en competencias consideradas básicas por actores clave del sistema jurídico. Si se atiende a esa suerte de consenso que ha acompañado a las quejas antes vistas, se percibe que, en la medida en que dicho consenso gana adeptos, exige de los demás actores una actitud reflexión, pero también de acción.

A su turno, como se ha observado del debate antes descrito, las propuestas de solución han apuntado hacia el diseño de un examen nacional, voluntario y a cargo de las universidades interesadas en él. Con todo, no parece que haya existido mayor avance en su elaboración, y es posible que ello se deba a varias razones. Entre las posibles, conjeturo que una iniciativa de esta naturaleza debería estar apoyada en un cierto corpus de literatura científica apropiada, así

\footnotetext{
${ }^{10}$ No es esta la ocasión para discutir acerca de qué debe o qué no debe considerarse competencia. Por lo pronto, sea suficiente, aunque provisional, la visión de la misma como una tríada compuesta por elementos cognitivos, procedimentales y actitudinales. Para una revisión de los conceptos de competencia, puede verse Goñı, María; Meseguer, Silvia, "Diseño Curricular centrado en las competencias que debe adquirir el estudiante del grado en Derecho", Formación Universitaria V. 3 No 2, 2010, pp. 37-46.
} 
como de una visión amplia y muy documentada de las experiencias comparadas, que hoy parece no existir, por lo menos, en las revistas jurídicas nacionales que debieran servir de cauce a este debate. Todo ello vuelve más difícil la elaboración del examen.

Así descrito, creo que es posible distinguir dos aspectos del problema:

a) El que se refiere a la toma de posición acerca de lo que se debe enseñar -qué conocimientos, cuáles destrezas-, cuestión a la que debe dar respuesta el plan de estudios. Sin una definición en este sentido, sería difícil contar con criterios que permitieran enjuiciar con cierta imparcialidad a las diferentes licenciaturas que se ofrecen hoy en el mercado de la educación.

b) El que se refiere a la forma en que se desarrolla el proceso enseñanzaaprendizaje del Derecho. Hasta el momento, se opera con un razonamiento bastante sencillo, que consiste en estimar que si los resultados son deficientes, la causa se encuentra en una inadecuada formación. Con todo, este juicio importa una evaluación del producto y no del proceso y, en tal sentido, es incompleta $\mathrm{o}$, si se prefiere, insuficiente.

La respuesta al primer aspecto del problema reenvía al de los criterios que guían la conformación de un plan de estudios. Si hasta hace pocos años no existían insumos provenientes más que de la percepción de los profesionales a cargo de los planes de estudio, hoy se cuenta con una mayor cantidad de literatura y estudios, los que, sin ser suficientes, pueden ayudar en la estrategia de la conformación de un plan de estudios innovador.

El segundo aspecto, más propiamente metodológico y objeto de este trabajo, se refiere a cómo enseñar conocimientos y desarrollar destrezas de manera tal que con ellos se garantice el desempeño profesional inicial de un recién titulado. ${ }^{11}$

\section{INSUMOS PARA LA DISCUSIÓN}

Si se tienen en cuenta las expresiones "competencias", "destrezas", "habilidades" que son utilizadas en el documento del Ministerio de Educación, es inevitable pensar como primer insumo los productos del llamado proceso de Bolonia y de lo que viene a ser un documento que, a su vera, se ha constituido en uno de los más importantes puntos de referencia. Me refiero al Informe Tuning para Latinoamérica, ${ }^{12}$ ampliación al ámbito latinoamericano de un estudio de pa-

\footnotetext{
${ }^{11}$ Suele entenderse por desempeño profesional inicial el de los primeros tres o cuatro años de la carrera. A partir de los siguientes, se entiende que el profesional adquiere y desarrolla otras destrezas, que probablemente no estuvieron en su formación inicial.

${ }^{12}$ Beneitone, Pablo; Esquetinl, César; González, Julia; Marty Maletá, Maida; Siufi, Gabriela; Wagenaar, Robert (Edits.), Reflexiones y perspectivas de la Educación Superior en América Latina. Informe Final Proyecto Tuning América Latina 2004-2007, Universidad de Deusto - Universidad de Groeningen, Bilbao, 2007. En: http://tuning.unideusto.org/tuningal/ [visitado el 26/01/2011].
} 
recidas características y de los mismos autores, centrado en el ámbito europeo. ${ }^{13}$ Se trata de un documento cuya lectura puede ser útil -pero no necesariamente decisiva- para dar inicio a la revisión de los problemas de educación jurídica en una perspectiva de formación basada en competencias, aun cuando algunos de sus resultados debieran ser objeto de una mejor discusión.

Otro insumo sin duda interesante lo constituye el documento Criterios de evaluación para la carrera de Derecho, de la Comisión Nacional de Acreditación. ${ }^{14}$ Este documento es particularmente relevante por cuanto sugiere un perfil de egreso que resulta ser el modelo conforme al cual se someten a acreditación todas las carreras de Derecho chilenas. Este documento no adopta la terminología propia de las competencias ni tampoco pretende elaborar un catálogo de éstas; sin embargo, constituye un material de obligada discusión, puesto que, no obstante carecer de toda sanción que le dé un carácter normativo general, impera de hecho a través de los procesos de acreditación de la carrera de Derecho.

Finalmente, hay que agregar los diferentes estudios que incluyen levantamientos de competencias, que algunas facultades de Derecho que han emprendido procesos de reforma curricular han tenido la ocasión de realizar. ${ }^{15}$

\section{Competencias, modalidades, métodos de enseÑanza Y EVALUACIÓN. ¿EJES DE LA RENOVACIÓN?}

Los resultados expuestos por el Ministerio de Educación pueden sugerir varias hipótesis destinadas a explicar el diagnóstico acerca de la deficiente formación de los profesionales del Derecho. Una hipótesis apuntaría a los métodos de enseñanza, es decir, suponer que, aunque sabemos qué debemos enseñar, no sabemos cómo se llega a producir el verdadero aprendizaje de los conocimientos necesarios para un buen desempeño. Otra hipótesis (dependiente o independiente de la anterior, según las premisas que se sigan) podría sugerir que las deficiencias se han de buscar en el nivel de conocimientos del profesorado en

${ }^{13}$ González, Julia ; WagenaAr, Robert (Edits.), Tuning Educational Structures in Europe. Informe Final Fase Uno, Universidad de Deusto - Universidad de Groeningen, Bilbao 2004.

${ }^{14}$ En: http://www.cnachile.cl/wp-content/uploads/2010/07/derecho1.pdf [visitado el 26/01/2011].

${ }^{15}$ Es el caso de las facultades de Derecho de la Universidad de Atacama y de la de Ciencias Jurídicas de la Universidad de Antofagasta, las que, en el marco del ya mencionado proyecto de rediseño curricular basado en competencias, MECESUP ATA 0706 ANF, permitió realizar un primer estudio acerca de las competencias más valoradas por jueces, abogados litigantes, egresados de la carrera, estudiantes, académicos y empleadores. El estudio realizado por el Prof. Dr. Adolfo González Brito puso de relieve que entre las competencias más valoradas, tomando en cuenta el conjunto de actores involucrados se encontraban las siguientes, en orden de prioridad: a) capacidad de razonar y argumentar jurídicamente; b) Actuar de manera leal, diligente y transparente en la defensa de los intereses de las personas a las que representa; c) capacidad de tomar decisiones jurídicas fundamentadas; d) capacidad para redactar textos y expresarse oralmente en un lenguaje fluido y técnico, usando términos jurídicos precisos y claros. 
sí, por lo que el resultado de los egresados de nuestras carreras no sería más que el reflejo de esa realidad. Y podríamos continuar. Sin embargo, puesto que carecemos de estudios empíricos y de información acerca de las variables involucradas, no estamos en condiciones de elegir una, ambas o, bien, desecharlas. De ahí que mi objetivo se limite sólo a proponer una discusión en torno a una de las variables: la idoneidad de los métodos de enseñanza.

Una observación rápida nos indica que en un porcentaje significativo de lecciones o clases de Derecho, la dinámica puede ser descrita como sigue: un docente desarrolla ante sus alumnos un discurso sobre la materia, en tanto que los discentes se limitan a tomar apuntes. En ocasiones, el profesor hace preguntas a sus alumnos y comprueba hasta qué punto éstos retienen lo que se ha dicho y en qué medida son capaces de razonar en cuanto a lo enseñado. Las evaluaciones, conforme a este patrón de enseñanza, evalúan la medida en que el alumno reproduce el conocimiento, a la vez que razona sobre él. ${ }^{16}$

Pero bastaría aguzar un poco la observación para descubrir que sería injusto sostener que los métodos de enseñanza del Derecho, en la actualidad, se limitan sólo a eso: la práctica de muchos docentes de Derecho integra también lecturas previas, trabajos de investigación, exposiciones, simulaciones, resolución de casos, debates organizados y así muchas otras iniciativas que deben valorarse. ${ }^{17}$ Más aún, la idea según la cual la clase teórica o magistral tiene importantes limitaciones parece ser una convicción que varios docentes tienen desde mucho tiempo antes de que se diese lugar al debate. La pregunta podría ser más bien otra: ¿por qué estas nuevas formas de enseñanza no se han constituido en un modelo generalizado? Parece claro que, a pesar de las convicciones compartidas, los docentes no hemos sido capaces de dar forma definitiva a un modelo que incluya esta clase de innovaciones. De ser así, la pregunta se transforma en otra: ¿sobre qué bases debiésemos sentar un sistema de enseñanza, capaz de articular diferentes métodos dentro de un modelo que sea, a su vez, eficiente desde la necesidad formar profesionales que no presenten las deficiencias denunciadas? Es probable que uno de los problemas consista en la carencia de un marco teórico compartido, aceptado, capaz de sistematizar, generalizar y profundizar en estos métodos, de modo de dar con un modelo de enseñanza efectivo y validado.

\footnotetext{
${ }^{16}$ Una crítica a este método de enseñanza, aquí muy esquemáticamente descrito, puede verse en CoLOMA, Rodrigo, "El ocaso del profesor Binns. Un ensayo acerca de la enseñanza del derecho en Chile", lus et Praxis Año 11 N$^{\circ} 1$, 2005, pp. 133-172.

${ }^{17}$ Con todo, la literatura científica no ha recogido estas experiencias -en la carrera de Derecho, específicamente-, por lo que muchos de estos intentos aún permanecen ocultos para la mayoría y sólo llegan a ser conocidos por los relatos que los propios docentes hacen de ellos ante sus colegas.
} 


\section{¿Puede el modelo de las competencias constituirse en el marco teórico apropiado?}

Este modelo viene siendo adoptado por la mayoría de las universidades pertenecientes al Consejo de Rectores, en todo lo cual se revela clara sintonía con propuestas surgidas, en este sentido, desde el Ejecutivo ${ }^{18}$. Así, al día de hoy, varias universidades han definido un conjunto de competencias que deben ostentar, al finalizar sus estudios, todos los estudiantes de la misma, con independencia de la carrera de la que egresen. Se trata de las llamadas competencias genéricas o transversales y son producto de una observación sobre las características y desafíos que plantea la sociedad y el mercado laboral actuales. ${ }^{19}$ Sirvan de ejemplo las competencias de pensamiento crítico y solución de problemas. Seguidamente, cada licenciatura define sus competencias específicas, las que vienen en medida importante a desarrollar estas competencias genéricas, encuadrándolas en las características, naturaleza y problemas de la propia carrera. En este sentido, la información proporcionada por el entorno es fundamental. ${ }^{20}$ Doy ejemplo de algunas de ellas, entre varias otras que se han levantado en el último tiempo para la carrera de Derecho:
a) Capacidad de razonar y argumentar jurídicamente. ${ }^{21}$

\footnotetext{
${ }^{18}$ Me refiero al documento elaborado por el Centro de Medición de la Pontificia Universidad CATÓlica de Chile, "Percepción de la Calidad Actual de los Titulados y Graduados de la Educación Superior Chilena", 2008, p. 10. En: http://www.mineduc.cl/usuarios/1234/File/Publicaciones/Informes/3estudio_final_estudio_calidad_de_la_educacin_superior_mide_uc.pdf [visitado el 02/02/2011]: "Es en este marco, el enfoque de competencias emerge como una de las formas de resolver las nuevas demandas del mundo laboral".

${ }^{19}$ Vid. Goñl; MeseGuer, "Diseño Curricular", cit. nota n. 10, p. 38.

${ }^{20}$ González Brito, Adolfo, "Definición y validación del perfil de egreso para el nuevo Rediseño Curricular de las Carreras de Derecho de la Universidad de Atacama y Antofagasta. Documento de trabajo", 2009, p. 3 sostiene, con referencia a la carrera de Derecho que: "fundamento de ello es que el mercado de los servicios profesionales de los egresados se desarrolla en un sistema jurídico dotado de normas de validez en todo el territorio nacional, teniendo en cuenta la apertura de mercados de trabajo internacionales y la posibilidad que proporciona el medio nacional a profesionales foráneos".

${ }^{21}$ Vid. Comisión Nacional de ACReditación de Chile, Criterios de evaluación para carreras de Derecho, p. 5. En: http://www.cnachile.cl/wp-content/uploads/2010/07/derecho1.pdf [visitado el 26/11/2011]; WagenaAr y Otros, "Informe Tuning América Latina" cit. n. 12, p. 123; y González, "Definición y validación", cit. nota n. 20, p. 7. Por su parte, GOÑI / MESEGUER, "Diseño Curricular", cit. n. 11, p. 42, concluyen para España una especial valoración de dos competencias que coinciden con la que analizamos, a saber, capacidad de análisis y síntesis y capacidad de argumentación y convicción. Pero sería justo agregar que éstos sólo vienen a corroborar una convicción arraigada, tanto en la práctica docente, como en la tradición jurídica occidental. No debe perderse que algunas reflexiones acerca de las destrezas del jurista son tan antiguas como la ciencia jurídica. Sobre la enseñanza del Derecho en la Edad Media, vid. WIELING, Hans, "Juristenausbildung im Mittelalter", en Baldus, Christian / FinKENAUER, Thomas y RüFNER, Thomas, Juristenausbildung in Europa zwischen Tradition und Reform, Tübingen, Mohr Siebeck, pp. 47-60 y BrundaGe, Thomas, The Medieval Origins of the Legal Profession, Chicago - London, The University of Chicago Press, 2008, pp. 219 y ss.
} 
b) Ser consciente de la dimensión ética de la profesión jurídica y de la responsabilidad social del graduado en Derecho y actuar en consecuencia. ${ }^{22}$

c) Capacidad para redactar textos y expresarse oralmente en un lenguaje fluido y técnico, usando términos jurídicos precisos y claros. ${ }^{23}$

d) Conocimiento sistemático de los principios generales e instituciones esenciales del Ordenamiento Jurídico. ${ }^{24}$

A su turno, la identificación de las competencias lleva, como es lógico, a la reflexión acerca del cómo se han de desarrollar aquéllas, lo que nos coloca frente al problema de la modalidad y del método de enseñanza. El concepto de modalidad de enseñanza es más amplio que el de método, puesto que remite a la forma de organización de los procesos de enseñanza-aprendizaje, cuyos ejes son los propósitos del docente y los recursos de la institución universitaria. Las modalidades proporcionan un marco muy amplio de actuación, dentro del cual pueden desenvolverse los métodos. Suelen reconocerse como modalidades habituales las lecciones teóricas, los seminarios o talleres, las clases prácticas, las prácticas externas, las tutorías, el trabajo en grupo y el trabajo autónomo. ${ }^{25}$ En la práctica docente tradicional, se reconoce como prevalente la modalidad de clases teóricas, intercaladas con un número variable de clases prácticas. A su turno, la asignatura de Clínicas Jurídicas, dependiendo de su orientación hacia

${ }^{22}$ Wagenaar et. al, "Informe Tuning América Latina" cit. n. 13, p. 123; González Brito, "Definición y validación", cit. n. 21, p. 7. En Comisión NaCiOnAl de ACReditACIÓN DE Chile, cit. n. 22, p. 5, se destaca la capacidad que el egresado de Derecho debe tener para ser capaz de "discernir y hacer valoraciones éticas con relación a normas o situaciones específicas y frente a posiciones o soluciones jurídicas"; GoÑl; Meseguer, "Diseño Curricular", cit. nota n. 10, p. 42, destacan el compromiso ético, para el cual exigen que se enseñe (p. 44) "no como asignatura, sino impregnando cada una de las ramas del saber y en cada actuación y enseñanza impartida". Puede observarse que la alta valoración que tiene esta competencia coincide con y se refleja en la preocupación del exministro Marín por los riesgos que acechaban a la ética profesional.

${ }^{23}$ Comisión Nacional de Acreditación de Chile, cit. n. 22, p. 5; Wagenaar et. al, "Informe Tuning América Latina" cit. n. 13, p. 123; GonZÁlez Brito, "Definición y validación", cit. n. 21, p. 7. Por su parte GoÑl; MESEGUER, "Diseño Curricular", cit. nota n. 10, p. 42 sólo consideran los conocimientos como parte de la competencia relativa a la capacidad de resolución de problemas y no de forma autónoma, lo que parece coherente con la propia definición de competencia uno de cuyos elementos es el conocimiento. Las autoras coinciden con la afirmación según la cual "no se trata sólo de adquirir conocimientos sino de saber qué se hace con ellos" (p. 39).

${ }^{24}$ Comisión Nacional de Acreditación de Chile, cit. n. 22, p. 5; Wagenaar et. al, "Informe Tuning América Latina" cit. n. 13, p. 123; GonZález Brito, "Definición y validación", cit. n. 21, p. 7. Sólo el documento de la Comisión Nacional de ACreditación De Chile, cit. n. 22, va más allá y expresa que las principales áreas del conocimiento sobre las que se debe expresar este conocimiento son: Derecho Civil, Derecho Constitucional, Derecho Penal y Derecho Procesal.

${ }^{25}$ Una descripción de estas modalidades de enseñanza, en De Miguel Díaz, Mario, Modalidades de enseñanza centradas en el desarrollo de competencias, Universidad de Oviedo, Oviedo, 2006, pp. 50 y ss. 
casos reales o ficticios, constituyen un buen ejemplo de prácticas externas o talleres, según el caso.

Despejada la definición de la modalidad, comparece a continuación el de la elección de los métodos de enseñanza, los que tienden a insertarse en la primera, una vez que existe claridad acerca de la competencia a desarrollar y su nivel de desarrollo. Los métodos de enseñanza que suelen identificarse como prácticas habituales son, genéricamente, los siguientes: lección magistral, estudio de casos, resolución de problemas, aprendizaje basado en problemas, aprendizaje basado en proyectos, aprendizaje cooperativo y contrato de aprendizaje. Sobre la lección magistral, no es necesario decir mucho, puesto que pareciera tratarse del método prevalente, a pesar de que la información empírica sobre métodos de enseñanza es, en nuestro medio, exigua. ${ }^{26}$

En la formación jurídica actual, el método de la lección magistral parece ir acompañado ahora por actividades diferentes, que proponen al estudiante problemas o situaciones, para las que debe hacer uso de un mayor caudal de recursos, no sólo de conocimientos, sino también actitudinales. Así, se observa cada vez más la existencia de sesiones dedicadas al estudio analítico de jurisprudencia (lo que inequívocamente corresponde al método de estudio de casos), la investigación monográfica (que, dependiendo de las características que le imprima el docente, constituyen ejemplos de resolución de problemas o aprendizaje basado en problemas), las pasantías en tribunales, fiscalías o defensorías (que podría identificarse como un aprendizaje basado en proyectos, en la medida en que la pasantía tuviese una organización adecuada). Estas prácticas enriquecen el acervo de recursos didácticos, pero lo que se requiere es que su puesta en práctica esté en consonancia con la competencia a cuyo desarrollo se quiere contribuir, de modo de poder ajustar la práctica docente a ese objetivo.

A primera vista, pareciera que el problema del método está solucionado in nuce una vez que se ha decidido la competencia a desarrollar. Sin embargo, esta

\footnotetext{
${ }^{26}$ Por lo pronto, de los resultados de los procesos de acreditación se puede observar que la regla general es que los métodos de enseñanza no sean objeto de evaluación. Cfr. Comisión NaCIONAL DE ACREDITACIÓN De Pregrado, Acuerdo de Acreditación No 300, de 26/07/2006, p. 3, En: http://www.cnachile.cl/wpcontent/uploads/2010/08/ACUERDO_N300.pdf [visitado el 05/03/2011], que se limita a constatar, sin emitir juicios de valor, que "la metodología utilizada es tradicional, centrada principalmente en clases magistrales..."; Agencia Acreditadora de Chile, Acuerdo de Acreditación No 32, de 07/07/2010, p. 3. En: http://www.cnachile.cl/wp-content/uploads/2010/08/acuerdo_32_derecho_ucsc1.pdf [visitado el 05/03/2011], que vuelve a constatar la existencia de clases magistrales como el método predominante. De mayor interés resulta el documento Comisión Nacional de Acreditación de Pregrado, Acuerdo de Acreditación No 251, de 10/01/2006, p. 3. En: http://www.cnachile.cl/wp-content/uploads/2010/12/ ACUERDO_N301.pdf [visitado el 05/03/2011], que destaca como fortaleza de la carrera examinada "la introducción de nuevos métodos activos y participativos, con un éxito todavía parcial, lo que puede tener como consecuencia algunas inconsistencias metodológicas entre asignaturas o secciones de las mismas".
} 
conclusión no es del todo acertada. Por una parte, es menester tener presente que las competencias se desarrollan a lo largo de todo un plan de estudios, razón por la cual ninguna asignatura, por regla general, desarrolla una competencia por completo, sino de un modo gradual. Además, no se puede dejar de lado el aprendizaje de los conceptos fundamentales de la disciplina, lo que remite a métodos tradicionales. De este modo, se tiende a la mixtura de métodos; éstos podrían ser, por ejemplo, uno o varios ejercicios de redacciones de documentos de la práctica jurídica (resolución de problemas), al mismo tiempo que, para la adquisición sistemática de conocimientos, podrían concurrir la clase magistral y el estudio individual. Hay que poner de relieve, eso sí, que no se trata de algo que pueda quedar librado al azar. Las diferentes modalidades se relacionan en distinto grado de intensidad con los métodos y así, por ejemplo, sabemos que -desde una perspectiva general y no centrada específicamente en la carrera de Derecho- mientras la modalidad de enseñanza consistente en clases teóricas tiene menos posibilidades de albergar otros métodos, la que disfruta de una mayor flexibilidad y cobertura es la del trabajo en grupos, el estudio individual y la tutoría. ${ }^{27}$

Finalmente, el último eslabón de esta cadena es la evaluación. La coherencia que debe observar ésta con los estadios anteriores ha de ser máxima, puesto que, a diferencia de la forma más habitual de pregunta-respuesta, el proceso evaluativo debe ser capaz de entregar información al docente acerca de todas las destrezas del estudiante, no sólo la de reproducción memorística. ${ }^{28}$

Desde luego, tanto la elección del método, como de la modalidad, como de la evaluación a adoptar son aspectos fundamentales de la práctica docente. Contribuye mucho al éxito de la elección de cada uno de estos eslabones y a su correcta ejecución, un cierto grado de dominio de técnicas de pedagogía aplicada a la educación universitaria, lo que bien puede lograrse a través de cursos de perfeccionamiento para docentes.

\section{UN CASO IMAGINARIO}

Permítaseme, para concluir, imaginar los perfiles básicos del diseño de un asignatura de perfil teórico, correspondiente a los primeros dos años de estudios.

\footnotetext{
${ }^{27}$ Es el resultado al que llega De Mıguel Díaz, Mario, Modalidades de enseñanza, cit. n. 26, p. 112, a través de una tabla de doble entrada, en que se observa la relación modalidad/método. Este resultado debe ser, a la vez, puesto en relación con el que analiza la relación entre método y desarrollo de competencia, en los que destacan el aprendizaje basado en problemas, la resolución de problemas y el aprendizaje cooperativo (p. 113).

${ }^{28}$ Sobre la evaluación y el desarrollo de competencias, con algunos ejemplos, vid. DelGado García, Ana María (Coord.); Borge Bravo, Rosa; García Albero, Jordi; Oliver Cuello, Rafael; Salomón Sancho, Lourdes, Competencias y diseño de la evaluación continua y final en el Espacio Europeo de la Educación Superior, Ministerio de Educación y Ciencia - Dirección General de Universidades, Madrid, España. En: http://campus.usal.es/ ofeees/ARTICULOS/competencias_evaluacion_eees_mec.pdf [visitado el 02/02/2011].
} 
Pienso en asignaturas tales como Derecho romano, Introducción al Derecho o Derecho político, según la nomenclatura tradicional. Asumiré que a esta asignatura se le exige desarrollar en un nivel básico la competencia relativa al razonamiento y argumentación jurídica. ${ }^{29}$ Me permitiré realizar una exposición breve -que podría no hacer justicia a la complejidad del proceso-, con la sola finalidad de indicar un esquema de actuación que permita columbrar algunos aspectos claves de la transformación.

a) Identificación de la competencia.- Un primer punto consistirá en la identificación de las competencias profesionales. El docente o su equipo podrán recurrir a los documentos e informes que antes he citado, ${ }^{30}$ en los que las competencias profesionales del abogado aparecen redactadas en términos bastante amplios. Así, por ejemplo, la información disponible nos indica que la competencia "Razonamiento y argumentación jurídica" es una de las más importantes. Puesto que una competencia no puede desarrollarse íntegramente en una asignatura, sino más bien en ciclos de formación, habrá que decidir el nivel en que se buscará su desarrollo. Y, puesto que he elegido una asignatura de los dos primeros años, la competencia podría quedar redactada en los siguientes términos:

En el contexto de un problema imaginario (un conflicto procesal, celebración o interpretación de un acto jurídico), el estudiante identifica los supuestos de hecho y las normas relativas al problema, y propone una solución al mismo, utilizando con propiedad el lenguaje técnico-jurídico, con miras a la búsqueda de una solución justa.

En la descripción es posible advertir la presencia de elementos procedimentales (análisis de variables, propuesta de solución) y los actitudinales (la orientación hacia la decisión justa). A su turno, los elementos cognitivos están supuestos en los procedimentales, puesto que son la base de las destrezas intelectuales en juego.

b) Estructura de contenidos, modalidades y métodos de enseñanza.- Los contenidos de las disciplinas más tradicionales requieren una revisión, para identificar elementos indispensables en la formación y otros que no tengan este carácter. Con todo, los alcances de la revisión podrían ser muy acotados, puesto que probablemente la mayoría comparecerá en la formación de un estudiante. Pero lo que requiere una atención especial es el problema relativo a

${ }^{29}$ Consignemos enseguida que el hecho de que una asignatura tenga encomendada el desarrollo de una competencia, no implica que no pueda desarrollar otras, lo que en ocasiones es inevitable. Significa, tan sólo, que el núcleo de su actividad está constituido por esa competencia, que es la que aparece con un mayor relieve frente a las demás.

${ }^{30}$ Idealmente, es mejor acometer un estudio empírico, con una amplia participación de actores del mercado laboral. Con todo, esta última es una solución que lleva tiempo y exige recursos que muchas veces no se tienen a la mano. 
las características de los contenidos, es decir, el carácter teórico o práctico de los mismos. Asumiré que en una asignatura de primer año, los contenidos son predominantemente teóricos, lo que ya nos da una idea acerca de la modalidad (clases téoricas y prácticas) y de los métodos de enseñanza a elegir (lección magistral, estudio de casos, resolución de problemas) ${ }^{31}$. Puesto que en este nivel la importancia de la correcta apropiación de los contenidos es alta, es necesario delimitar cuánto de este aprendizaje se espera que sea obtenido mediante la asistencia a clases y cuánto mediante el estudio autónomo (que se agrega a los métodos antes descritos). ${ }^{32}$ En este sentido, parece conveniente establecer un mecanismo que permita al docente asegurarse de que cada alumno avanza correctamente en la dirección perseguida. Este importante objetivo podría cumplirse eficientemente a través de la tutoría a grupos de alumnos, lo que exigirá que estas tutorías estén adecuadamente planificadas. Así, la tutoría, se suma a los métodos a emplear.

c) Evaluaciones. Para comprobar que el estudiante ha adquirido la competencia, el docente deberá utilizar evaluaciones que le permitan revisar el proceso de adquisición de la competencia, es decir, que le permitan formarse una idea muy precisa acerca de que el estudiante está en condiciones de hacer precisamente lo que la definición de la misma dice que puede hacer. Lo normal será, pues, que a lo largo de varios ejercicios el estudiante se enfrente a desafíos en que se describan situaciones procesales o actos jurídicos a tal punto problemáticos, que la solución del problema sólo aparecerá con claridad después de un minucioso análisis del caso. Las herramientas evaluativas de las que se deberá disponer, por consiguiente, irán dando forma a una serie de trabajos que permitirán formarse una idea de lo preparado que está para resolver estas cuestiones, dando forma a un portafolio.

Desde luego, en un contexto como el explicitado, las evaluaciones son constantes, no siempre cuantitativas y nunca globales. Las conocidas pruebas departamentales o de cátedra, consistentes en extensos desarrollos, no deberían ser parte del repertorio evaluativo. Ello implica diseñar una tabla de puntuaciones acumulativas, que al final del proceso sean convertibles en notas.

Para cada evaluación, particularmente las que suponen solución de casos, el docente deberá contar con rúbricas que sean conocidas desde antes por el

\footnotetext{
${ }^{31}$ Aunque se refiere a una asignatura que se enseña en niveles superiores, es significativa la reflexión sobre la experiencia en la aplicación del método de casos de VARGAS VASSEROT, Carlos, "El método del caso en la enseñanza del Derecho: experiencia piloto de un piloto novel", Revista de Formación e Innovación Educativa Universitaria V. 2, № 4, 2009, pp. 193-206. En: http://webs.uvigo.es/refiedu [visitado el 02/02/2011.

${ }^{32}$ Un cálculo aproximado sería que por cada hora de lección magistral, se deba pensar en tres horas de estudio autónomo.
} 
alumno, en las cuales pudiesen ser expuestos los criterios conforme a los cuales se entienden aprobados los estándares exigidos. Ciertamente, la creación de estas evaluaciones y sus correspondientes rúbricas exigirán del docente horas de preparación que excederán con mucho, las habituales para una lección magistral en el contexto de una evaluación global y sumativa.

\section{ConClusiones}

El debate acerca de la formación de los abogados ha de ser objeto de un tratamiento científico y profundo, para lo cual es necesario, en primer lugar, contar con un marco teórico que permita homologar los términos y dotar de consistencia científica al debate. En este trabajo, me he referido al modelo de las competencias, modelo cuyos puntos de vista pueden y deben ser objeto de discusión a propósito de la formación jurídica, como lo son actualmente en la formación en otras áreas profesionales. Se trata de un debate abierto, con mucho campo para la discusión. En este contexto, los estudios que han intentado identificar estas competencias no han sido objeto de mayor discusión por parte de aquellos que asumen la dirección de instituciones educacionales ni, en general, por el mundo académico del Derecho. Por el contrario, en la discusión pareciera que se tiende a ignorar el conocimiento disponible y a formular ideas que carecen, por el momento, de una base teórica consistente y que sólo apelan a la imitación de ejemplos o modelos extranjeros. Motivación principal de este deseable debate debiera serlo la relevancia social que tiene la formación jurídica, como lo demuestra la preocupación de ciertos actores del mundo del derecho, que observan y evalúan el desempeño de los abogados y su impacto social. 
